\title{
SEXISMO EN EDUCACIÓN PREESCOLAR: LA PERSPECTIVA DOCENTE
}

\author{
Corrales Mejías, Lizeth ${ }^{1}$, Delgado León, María Isabel ${ }^{2}$, \\ Herrera Valverde, Carolina ${ }^{3}$, Pereira Pérez, Zulay ${ }^{4}$, \\ Sancho Chavarría, Jeannette ${ }^{5}$, Zamora Salas, Mariela ${ }^{6}$
}

Resumen

En la sociedad actual, es evidente encontrar elementos sexistas que discriminen, principalmente, a las mujeres. Estereotipos y creencias influyen en este fenómeno. En este artículo, se hace un estudio de las opiniones que tienen las docentes de preescolar acerca de esta temática y se concluye que han logrado observar diferencias entre las actividades que realizan los niños y las niñas, pues se nota una separación y tales conductas obedecen a lo aprendido en sus hogares.
Abstract

In current society it is impossible not to find sexist elements that discriminate especially women. Stereotypes and beliefs influence this phenomenon. This article studies the opinions held by preschool teachers on this matter. The conclusion is that these teachers have observed differences in the activities boys and girls do, and that these behaviors are learned in their homes.

Descriptores: sexismo, género, educación preescolar, papeles sexuales, estereotipos sexuales, opiniones de docentes.

\footnotetext{
${ }^{1}$ Corrales Mejías, Lizeth. Licenciada en Educación Preescolar. Docente de Preescolar. Actualmente se desempeña en el área de Educación. tiene publicaciones en el área de educación ambiental y Pedagogía.

2 Delgado León, María Isabel. Licenciada en Educación Preescolar. Docente de Preescolar. Actualmente se desempeña en el área de Educación. tiene publicaciones en el área de educación ambiental y Pedagogía.

${ }^{3}$ Herrera Valverde, Carolina, Licenciada en Educación Preescolar. Docente de Preescolar. Actualmente se desempeña en el área de Educación, tiene publicaciones en el área de educación ambiental y Pedagogía.

${ }^{4}$ Pereira Pérez, Zulay, Catedrática de la Universidad Nacional. Máster en Psicología de la Universidad de Costa Rica. Ex Coordinadora e Investigadora del Programa de Investigación en Epistemología Genética y Educación del IIMEC. Universidad de Costa Rica. Ex Directora de la División de Educación Básica del CIDE-UNA. Actualmente se desempeña como docente e investigadora en el Centro de Investigación y Docencia en Educación. Tiene publicaciones en el campo de la Psicología y la Pedagogía.

${ }^{5}$ Sancho Chavarría, Jeannette, Licenciada en Educación Preescolar. Docente de Preescolar. Actualmente se desempeña en el área de Educación. tiene publicaciones en el área de educación ambiental y Pedagogía

${ }^{6}$ Zamora Salas, Mariela, Licenciada en Educación Preescolar. Docente de Preescolar. Actualmente se desempeña en el área de Educación, tiene publicaciones en el área de educación ambiental y Pedagogía.
} 

ablar de sexismo es entrar en un amplio andamiaje de concepciones, debido a la polémica que causa dicho tema. Roles, estereotipos y creencias son solo algunos conceptos ligados a la temática que se enlazan entre sí.

El concepto de sexismo posee varias definiciones, creadas a la luz de diferentes aristas poseedoras de un sinnúmero de creencias propias de persona. El sexismo como tal no surge como una moda, por el contrario es algo que ha permanecido latente en la cultura en el transcurso del tiempo. Es importante recalcar que cada cultura lo manifiesta, transmite y vivencia de una forma específica, relacionado por supuesto, con sus tradiciones y modo de vida propio.

En relación con el sexismo, Lamas (citado por Scala, 2001) señala que “(...) sexismo es la expresión más negativa y violenta del esquema cultural de género" (p. 19). La afirmación anterior responde a la discriminación que se hace palpable a uno de los dos sexos, femenino o masculino. Sin embargo, en la sociedad pasada y actual se ha hecho evidente con mayor fuerza en contra de las mujeres.

Sobre este aspecto, la autora antes mencionada propone otra definición, más específica y directa; dice que "sexismo sería la suma del poder político, social y cultural en los varones” (p. 20). Dicha afirmación se vislumbra en tomo a una sociedad clasista, con roles y papeles ya distribuidos no por potencial o valía del ser humano, sino por tradiciones y prejuicios predeterminados.

Podría decirse que el sexismo es una construcción social, que lleva consigo toda una historia de formación en la que han influido significativamente la religión, escuela, aspectos fisiológicos, psicológicos, entre otros. Estos factores han sido fortalecidos y reforzados en muchas ocasiones por las familias.

El tema del sexismo trasciende cada ámbito de la vida del ser humano. Se encuentra tan marcado en el ámbito social, cultural, religioso, político, científico, entre otros que hasta se ha fusionado en el sistema educativo, propiamente en la edad preescolar, donde se vislumbran algunos comportamientos que perfilan la personalidad de los niños y las niñas, primero en el marco familiar y luego en forma conjunta con el centro educativo.

La importancia de trabajar este tema con niñas y niños preescolares de 5 a 6 años, se deriva de la necesidad de conocer todas aquellas prácticas sexistas que desde su percepción se tienden a reforzar, así como a reproducir en el aula. Al profundizar en el tema del sexismo y la perspectiva que tienen los niños y las niñas, hay más posibilidad de que se eviten distinciones de sexo, porque se empieza a crear en el y la infante la capacidad de reconocer las situaciones que permiten convivir en una sociedad justa. Esto podrá lograrse siempre y cuando en el sistema educativo se fomente un ambiente que vaya en pro de la equidad de género. 
Debe recordarse que algunas formas, por las cuales el niño o la niña construye su conocimiento, son la interacción con el medio y el proceso de imitación. Este aprendizaje mucho dependerá del medio familiar, social, cultural, que se le brinde, con el fin de cambiar, poco a poco, la visión sexista que tiene la sociedad actualmente.

En la etapa preescolar, el infante desarrolla muchas destrezas y habilidades importantes para lograr un buen equilibrio en las etapas posteriores del crecimiento y desarrollo. En ella, tanto el niño como la niña entran de lleno e incursionan en una multiplicidad de ambientes, hasta ahora asumidos sólo por el círculo familiar; así, el contacto con un centro educativo les provocará un conflicto al experimentar y conocer realidades muy diferentes, pero congruentes con el objetivo de brindar la mejor educación posible para su desenvolvimiento dentro de su entorno inmediato.

Esta es una tarea muy difícil, porque los niños y las niñas imitan lo que los adultos hacen y al ingresar a la educación preescolar, ya traen un cúmulo de experiencias que, en una mayoría, podrían estar dirigidas a conductas sexistas. Debe aclararse que estos comportamientos sexistas, en ocasiones, se transmiten en forma inconsciente por parte del núcleo familiar, es decir, por falta de información y además por aspectos propiamente culturales contemplando, según Macroby (citado por Castro, 1999), “(...) la rigidez de los roles implantados en la sociedad limitan las potencialidades de los seres humanos” (p. 4).

En resumen, puede decirse que al estar inmersos en una sociedad sexista, los adultos y las adultas asumen comportamientos dirigidos al sexismo directamente y, por consiguiente, los y las infantes siguen estos pasos. En este sentido, es interesante retomar el análisis de este tema desde la perspectiva infantil.

No se pretende, en ningún momento, borrar lo aprendido por los niños y niñas durante su desarrollo; simplemente, se trata de que conozcan otros aprendizajes para que se comporten de otra forma y crezcan como personas libres de prejuicios.

El sexismo en la educación se ha dado por mucho tiempo y la investigación realizada muestra un panorama de lo que se ha venido vivenciando, así como de la perspectiva del tema y de los roles sexuales que tienen los niños y las niñas que cursan el nivel preescolar en instituciones educativas costarricenses.

En preescolar, se hace necesario desarrollar en el párvulo y la párvula, una percepción del mundo en que vive, donde actualmente se lucha por la igualdad de género y de oportunidades. Al rescatar la perspectiva del niño y la niña a cortas edades y al adentrarse en las concepciones que tienen las docentes que los atienden en ese nivel educativo, se obtendrá un mayor conocimiento de lo que sucede en las aulas y se comprenderá mejor aquello que deba ser modificado 
en la sociedad, para evitar de esa manera, la discriminación, tanto del género femenino como del masculino.

El género hay que entenderlo como algo social y no biológico, ya que su génesis radica en bases culturales establecidas. Las constantes interacciones sociales mediante los diferentes sectores entendidos como la familia, la religión, la educación y la misma cultura provocan, en la sociedad, un condicionamiento que genera comportamientos aceptados o rechazados en el modo de vida imperante.

El proceso de socialización responde de manera significativa a un continuo aprendizaje de conductas y de normas que caracterizan a las personas como masculinas o femeninas. Al respecto, Sánchez (citado por Castro, 1999) considera que “(...) la socialización se convierte en el modelo de nuestra cultura en la medida en que es una construcción artificial que responde a los estereotipos, a los comportamientos socialmente esperados" (p. 2). Como ser social, el ser humano, durante toda su vida, desarrolla y estructura relaciones con el ambiente en el que se desenvuelve y con sus iguales; no obstante este fenómeno sucede a ritmos particulares y de diferente intensidad, según sea el proceso de crecimiento del individuo. Así, puede entenderse por qué la identidad del género que se estructure, ya sea de un niño o de una niña, dependerá, entre otros factores, de acciones sociales como los valores, las representaciones positivas y negativas de cada sexo, así como el contexto histórico en el cual se ve enmarcado (Ayales, Benítez, Fevrier y Ramírez, s.f.).

Entendida la socialización como un puente de transmisión de conductas para con el género, es fácil determinar su relevancia de generación en generación. El autor Martín-Baró (citado por Ayales, et al., s.f.) señala que los seres humanos adquieren una identidad personal y social a raíz de este proceso de socialización constante durante toda su vida.

En la sociedad costarricense desde tiempos antiguos, los patrones de crianza, según las responsables de la presente investigación, se han vislumbrado en tres órdenes: uno estático, que se mantiene de generación en generación en relación con la transmisión de roles; otro, que puede entenderse como ideológico en el sentido de que el modelo transmitido tiene una ideología ya definida; y el último, un orden práctico el cual combina los anteriores. Estos órdenes, si se quiere, forman parte fundamental de la cultura costarricense, las personas son criadas dentro de los mismos.

Según Lagarde (1999), cada persona “(...) asume dichas particularidades como consecuencia de aspectos étnicos, raciales, de clase, de edad, salud, religiosas, políticas, ideológicas y culturales" (p. 133). El cúmulo de estas consecuencias repercute en su concepto de la persona. 
Los elementos positivos o negativos que constituyen este fenómeno influyen de manera directa en el significado del término género; los conceptos atesorados por la tradición, se ven transformados por las variaciones que presentan en la cultura y en sus manifestaciones.

Con el transcurrir del tiempo, las concepciones e ideologías varían. Cada vez es más palpable la adopción de modelos de vida ajenos, principalmente norteamericanos y europeos, en donde la libertad de conducirse por la vida y el despojo de valores arraigados se hace notable.

Sin embargo, los y las costarricenses mantienen muy arraigados los valores, costumbres y tradiciones transmitidos por sus antecesores. Por ello, se han transmitido modelos específicos de conducta y de roles, en donde el hombre tiene el poder, la fuerza, la valentía y entereza y hasta la inteligencia. La mujer, por otra parte, tiene un papel de servicio, sumisión, delicade-
El concepto de sexismo posee varias definiciones, creadas a la luz de diferentes aristas poseedoras de un sin número de creencias propias de persona. El sexismo como tal no surge como una moda, por el contrario es algo que ha permanecido latente en la cultura en el transcurso del tiempo. Es importante recalcar que cada cultura lo manifiesta, transmite y vivencia de una forma específica, relacionado por supuesto, con sus tradiciones y modo de vida propio.

za y abnegación; según Castro (1999), la función primordial y social de la mujer “(...) resulta principalmente la de ser madre y por ello, se especializa totalmente en la esfera reproductora y del trabajo doméstico, como una esfera aislada de las demás relaciones sociales" (p. 11). Tal como lo señala Ayales et al. (s.f.).

(...) el modelo familiar de los países centroamericanos responde de cierta manera a su estructuración por factores de índole económica, social y política; lo cual ha repercutido en el accionar de la constitución de patrones de crianza, autoridad y desarrollo personal (p. 19).

Se tiene una visión optimista de la concepción de género, al brindarse un espacio activo a la mujer dentro de la sociedad, pues se denota un despojo de prejuicios tradicionales. Se quiere una sociedad más equitativa y certera, en 
donde tanto hombres como mujeres sean valorados por sus capacidades y no por la determinación y asignación social de una condición a la que se ven sometidos desde que nacen: ser hombre o mujer.

Dentro de cada una de las sociedades, las personas establecen relaciones e interiorizan una manera de comportarse o actuar de acuerdo con el contexto en el que están inmersos; pero este a su vez responde directamente a los patrones culturales imperantes en esa sociedad. Muchos autores han realizado estudios y han concluido que el aprendizaje social que experimenta cada persona es determinante; porque de una u otra forma, son los referentes que sujeto utiliza como punto de partida para basar los comportamientos propios de cada momento de la vida.

A través de la historia, se hace evidente que los hombres y las mujeres han recibido todo un bagaje cultural, transmitido por medio de los procesos sociales en los que han estado inmersos. Baró (citado por Quirós, 1989) señala que dichos procesos, pueden darse de diferentes maneras, entre las cuales menciona las relaciones primarias, relaciones funcionales y relaciones estructurales.

Según con lo anterior, puede decirse que el legado cultural y social que es transmitido de generación en generación se vale de la familia inicialmente, la escuela en segundo plano y de la sociedad como referente en ambos casos, para conservar y perpetuar las ideologías que predominan en las diferentes culturas. Estos procesos son los que han conducido a las personas a asumir y desarrollar determinadas funciones, que no han sido las mismas para todas; es aquí donde el sexo empieza a jugar un papel determinante dentro de las dinámicas de las sociedades; los quehaceres que se le asignaban a las personas eran correspondientes con su sexo, por ello, es que se habla de roles sexuales. Tal y como señala Luna (2003) este fenómeno sucede, porque

(...) durante muchos años se trató la masculinidad y la feminidad como una dimensión única, con dos polos opuestos, (...) es decir, estos podrían ser en mayor o menor grado masculinos o femeninos, pero nunca ser ambas cosas a la vez" (p. 29).

Cabe destacar, que en todo este juego de relaciones, la familia se encarga de inculcar en sus miembros diferentes concepciones que, posteriormente, son asumidas como roles inquebrantables y además reforzados por el medio que la rodea. De esta forma, por mucho tiempo, se ha pensado que todas las respuestas conductuales de los seres humanos están necesariamente influenciada por el medio; puede decirse que los roles sexuales hacen referencia a características de la personalidad, actitudes y conductas que cada cultura atribuye a los sexos; 
por lo tanto, existe una manera para modelar y premiar a aquellos que cumplen con lo que ha sido estipulado como masculinidad para los hombres y feminidad para las mujeres.

Es necesario aclarar que lo asumido como masculinidad o feminidad, se ha filtrado socialmente desde tiempos antiguos y continúa haciéndose; es un proceso que, de manera casi invisible, ha logrado trascender y, además, varía de una cultura a otra. Esto puede verse reflejado en las mujeres occidentales que, en su mayoría, no permiten a sus esposos tener a otra mujer; mientras, las mujeres musulmanas aceptan a las otras esposas como parte de su destino.

Es necesario rescatar que en todos los procesos sociales desarrollados cotidianamente, los niños y las niñas van interiorizando normas, reglas, creencias, actitudes y valores que responden a la ideología imperante dentro del sistema en el cual se desenvuelven. Es así como asuntos tan sencillos como la condición laboral, el vestuario y el lenguaje se constituyen en referentes transmisores llenos de significado, los cuales generan las nociones y conceptos que más tarde realizan y para luego convertirse en los roles sexuales.

El sistema educativo, al participar de la formación de los individuos, tiene una importancia particular; sobre todo si se trata de la función de roles femeninos y masculinos en la inmersión a la cultura; precisamente, porque es la educación la que debe garantizar el adecuado desenvolvimiento de los individuos en la sociedad. De esta manera, tanto los y las docentes, los directores y las directoras y los libros de textos que usan, comienzan a dejar huellas e impresiones en las mentes de las y los infantes. Es así como desde pequeños, logran hacer una división casi abismal entre todo el quehacer de varones y de las mujeres. En este sentido, varios autores han realizado estudios concernientes al tema de la masculinidad y feminidad y la relación entre estos; en algunas de las cuales, se ha llegado a conclusiones bastante importantes. Al respecto, Scott y Garret (citados por González, (1990) concluyen que:

- El contacto con materiales sexistas puede aumentar las actitudes sexistas, especialmente a temprana edad.

- El contacto con materiales que destacan la igualdad, producen una disminución de actitudes sexistas.

Si lo último es realmente verdad, cobra un papel importante la labor que el o la docente juega en los procesos de enseñanza y aprendizaje; de ahí la necesidad de indagar en estas temáticas.

El sexismo es una construcción social que lleva consigo toda una historia de formación en la que han influido significativamente la religión, escuela, 
aspectos fisiológicos, psicológicos, entre otros. Elementos que han sido reforzados, en muchas ocasiones. Véase la siguiente afirmación

(...) las madres y los padres, además de ocuparse de las necesidades materiales y afectivas de sus hijas e hijos, se encargan de la educación, formación de hábitos y de la adquisición de normas de conducta. En la familia se asimilan las pautas de comportamiento, los modelos y valores de género.

Aunque los papeles productivos y reproductivos de las mujeres están cambiando, es en la familia donde se sigue transmitiendo un modelo que otorga y responsabiliza a la mujer en el ámbito de "lo privado/ doméstico" y al hombre de "lo público" (Arenas, et al., 2002, p. 12).

Es evidente, por lo tanto, que el sexismo se muestra en la sociedad de diversas maneras y que a pesar de los esfuerzos que muchas personas realizan para ir erradicándolo sigue presente aún en la actualidad.

La investigación que dio origen a este artículo, fue de carácter exploratorio, pues no existen otros que aborden el tema desde la perspectiva que se hizo en esta investigación, aunque sí hay algunos que lo hacen desde otras disciplinas.

De acuerdo con el planteamiento de Barrantes (2000), el estudio explorativo se define como "(...) una investigación que se realiza para obtener un primer conocimiento de una situación (...) por eso se dice que tiene un carácter provisional" (p. 64).

Para efecto del presente artículo, se enfatizará en la información aportada por las docentes; en un artículo anterior ${ }^{7}$, se analizaron los resultados encontra-dos desde la perspectiva de niños y niñas que cursaban educación preescolar. Como instrumento básico para la recolección de la información, se usó el cuestionario con la finalidad de conocer las manifestaciones de sexismo desarrolladas dentro del aula preescolar.

Se destacan, seguidamente, los aspectos más relevantes referidos al sexismo en el aula preescolar costarricense, bajo el entendido de que los resultados no son generalizables, sino que se circunscriben a las instituciones en las cuales se llevó a cabo el estudio; no obstante, son un indicador de lo que sucede en aulas preescolares del sistema educativo costarricense. Asimismo, se incursiona en el análisis de las opiniones de las docentes preescolares acerca del sexismo en las aulas de este nivel educativo.

Es importante señalar que las docentes entrevistadas (20), tienen categorías profesionales de $\mathrm{Kt} 2$ (3) y Kt3 (17). Los años de servicio van de 1 a 20; de

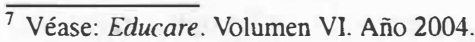


acuerdo con los intervalos, hay 4 docentes que han laborado de 1 a 5 años, 2 docentes de 6 a 10 años, 5 docentes de 11 a 15 años, 2 docentes de 16 a 20 años y 7 docentes han trabajado por más de 20 años.

La totalidad de las docentes manifiestan que entre sus estudiantes se dan diferencias por sexo. Igualmente, 17 docentes indican que en el jardín los niños y las niñas presentan conductas sexistas y 3 docentes dicen que no se presenta este tipo de conductas en su institución.

\section{Cuadro 1 \\ Concepción de género de las docentes entrevistadas.}

\begin{tabular}{lll}
\hline Tipo de respuesta & $F$ & $\%$ \\
\hline Diferenciación entre femenino y masculino. & 9 & 45 \\
Diferenciación entre sexos. & 7 & 35 \\
\hline
\end{tabular}

Fuente: Elaboración propia de las autoras.

En este cuadro, se recopilan las respuestas de las docentes acerca de ¿qué entiende por género? Es notable que la mayoría de docentes (9) da respuestas referidas a la diferencia entre lo femenino y lo masculino; por otra parte, ocupa una posición muy parecida la concepción acerca de diferenciación sexual (7 docentes) y diferenciación cultural, biológica y psicológica (4 docentes).

Es interesante observar como las docentes mencionan que el género responde a una diferenciación entre sexos, lo cual concuerda con lo que plantean Ayales et al. (s.f.) en el sentido que "Se refiere a las características sociales definidas por una sociedad determinada para normar el comportamiento, aptitudes, roles y funciones de mujeres y hombres y la manera que dicen relacionarse e interactuar" (p. 14).

\section{Cuadro 2}

Definición de roles sexuales o papeles, según opinión de docentes

\begin{tabular}{lcc}
\hline Tipo de respuesta & $F$ & $\%$ \\
\hline Comportamientos y conductas de cada género. & 13 & 65 \\
Patrón de conducta establecido por la sociedad. & 7 & 35 \\
\hline
\end{tabular}

Fuente: Elaboración propia de las autoras.

Ante la pregunta ¿Qué son roles o papeles sexuales?, las respuestas de las docentes se observan en el cuadro 2 y se dividen en dos categorías: la mayoría 
de docentes (13) opina que los papeles sexuales son comportamientos y conductas de cada género, sea este femenino o masculino. Mientras que las siete restantes, aseguran que los roles sexuales obedecen a un patrón de conducta establecido en la sociedad en la que se encuentra inmersa la persona.

Es notorio como ambas categorías coinciden en que la conducta juega un papel crucial en la constitución de los roles sexuales, independientemente si estos son un asunto personal de cada género o, por el contrario, una herencia social aprendida. Se dice que los roles sexuales están relacionados con el comportamiento de los seres humanos según el sexo al que pertenecen y son fortalecidos tanto en la familia, como en la escuela; de ese modo, se incorporan a la conducta de cada persona.

Las conceptualizaciones expresadas por las docentes coinciden con lo que las investigadoras a cargo de este estudio han definido como roles sexuales ${ }^{8}$, es decir, comportamientos de los seres humanos de acuerdo con el sexo (mujer o hombre) aceptados socialmente.

\section{Cuadro 3}

\section{Definición de sexismo según la opinión de las docentes entrevistadas.}

\begin{tabular}{lcc}
\hline Respuestas referidas & $F$ & $\%$ \\
\hline Actitudes discriminatorias hacia el género opuesto. & 9 & 45 \\
Diferenciación entre sexos. & 8 & 40 \\
Actividades que realiza cada sexo. & 3 & 15 \\
\hline
\end{tabular}

Fuente: Elaboración propia de las autoras.

El cuadro anterior hace referencia a la definición que dan las docentes de preescolar acerca de sexismo. Ante dicho cuestionamiento, las respuestas se situaron en tres categorías, dos de las cuales obtuvieron un porcentaje similar. En la primera categoría, nueve docentes coinciden al señalar que el sexismo responde a actividades discriminatorias hacia el género opuesto. Por otra parte, en la segunda, ocho docentes manifiestan que obedece a una diferenciación entre sexos y sólo tres docentes opinan que el sexismo se refiere a las actividades que realiza cada sexo.

Lo anterior reafirma la aseveración de González (1990) cuando dice que se denomina “....sexismo a todos aquellos procesos que limitan el desarrollo de las potencialidades esenciales debido al sexo de cada persona y por ende de su

$\overline{8}$ Véase glosario. 
grupo de iguales (...) el sexismo es la discriminación relacionada con la pertenencia a un sexo determinado" (p. 11).

\section{Cuadro 4 \\ Percepción que tienen las docentes en cuanto a los roles sexuales en la sociedad.}

\begin{tabular}{lcc}
\hline Respuestas referidas a que: & $F$ & $\%$ \\
\hline Se estimulan en la sociedad. & 14 & 70 \\
Cambian por la posición actual de la mujer. & 4 & 20 \\
Son adquiridos y transmitidos en la familia. & 2 & 10 \\
\hline
\end{tabular}

El cuadro anterior hace referencia a las opiniones de las docentes con respecto a los roles sexuales que hay en la sociedad; las respuestas priorizan mayoritariamente en tres aspectos: el primero menciona que los roles sexuales son estímulo de la sociedad (14 docentes), el segundo propone un cambio debido a la posición actual de la mujer (4 docentes), y el tercero manifiesta que es en el ámbito familiar en donde se adquieren y se transmiten los roles (2 docentes).

En dicho sentido, Macroby (citado por Castro, 1999) indica “(...) la rigidez de los roles implantados en la sociedad limitan las potencialidades de los seres humanos" (p. 4).

\section{Cuadro 5}

Percepción de estereotipos sexuales en la sociedad según las docentes entrevistadas.

\begin{tabular}{lcc}
\hline Respuestas que indican que: & $F$ & $\%$ \\
\hline Fomentan la desigualdad en hombres y mujeres. & 16 & 80 \\
Cambian por la posición actual de la mujer. & 3 & 15 \\
Posición de la Iglesia. & 1 & 5 \\
\hline
\end{tabular}

Según la percepción de las docentes en cuanto a los estereotipos sexuales en la sociedad, dieciséis opinan que estos fomentan la desigualdad entre mujeres y hombres; tres señalan que cambian por la posición actual de la mujer y una sostiene que la posición de la iglesia juega un papel importante. Es interesante anotar que la mayoría opina que los estereotipos sexuales son generadores de desigualdad, ya que minimizan o maximizan el sexo. Por otra parte, es importante señalar que desde la perspectiva de las investigadoras, los estereotipos sexuales generan discriminación basados en las creencias que existen diferencias entre el sexo femenino y masculino. 


\section{Cuadro 6}

Conductas sexistas que presentan niñas y niños, según la opinión de docentes.

\begin{tabular}{lll}
\hline Respuestas referidas a & $F$ & $\%$ \\
\hline Limitaciones de actuación producto & & \\
de la influencia del hogar. & 9 & 45 \\
Minimizar las capacidades de la mujer. & 3 & 15 \\
Juegos en el jardín. & 3 & 15 \\
Maximizar la capacidad del hombre. & 2 & 10 \\
No responde. & 3 & 15 \\
\hline
\end{tabular}

El cuadro 6 se refiere a ¿Cuáles son las conductas sexistas que presentan los niños y las niñas? Las opiniones de las docentes ante esta pregunta son variadas. Nueve docentes opinan que las conductas obedecen a limitaciones de actuación producto de la influencia del hogar; por otra parte, minimizan la capacidad de la mujer y los juegos en el jardín de niños. Para tres docentes, son conductas sexistas palpables. Otro grupo (2) señala que una conducta sexista es maximizar la capacidad del hombre, menospreciando así la capacidad femenina; por último, tres docentes no responden a esta pregunta.

Según los datos recopilados, las conductas citadas se dan tanto en el jardín infantil como en el hogar, producto de patrones culturales y modelos aprendidos de las personas adultas. En este sentido, Lamas (citado por Scala 2001) indica que “(...) el sexismo es la expresión más negativa y violenta del esquema cultural de género" (p. 19).

\section{Cuadro 7}

Manifestación de roles o papeles sexuales en la interacción entre niñas y niños según opinión de las docentes.

\begin{tabular}{lcc}
\hline Respuestas referidas a & $F$ & $\%$ \\
\hline $\begin{array}{l}\text { Diferencias de conducta de acuerdo con el género } \\
\text { (masculino-femenino) }\end{array}$ & 6 & 30 \\
Tipos de materiales y juegos que seleccionan. & 5 & 25 \\
Juego en áreas. & 5 & 25 \\
Juegos al aire libre. & 2 & 10 \\
Utilización del servicio sanitario. & 1 & 5 \\
En todo momento. & 1 & 5 \\
\hline
\end{tabular}

Fuente: Elaboración propia. 
En este cuadro, se hace referencia a la manifestación de roles o papeles sexuales en la interacción de niñas y niños. Las docentes entrevistadas dividen sus opiniones hacia dicha pregunta respondiendo una leve mayoría (6), que las diferencias de conducta, de acuerdo con el género, son las que mayoritariamente se presentan en la interacción entre niñas y niños. Cinco de ellas opinan que la manifestación de roles sexuales radica en el tipo de materiales y juegos que seleccionan las niñas y los niños. Con la misma frecuencia el juego en las áreas es manifestación de roles sexuales y los juegos al aire libre evidencian manifestaciones de roles sexuales, según dos docentes.

Se menciona también la utilización del servicio sanitario (1) y el hecho de que en todo momento se manifiestan los roles sexuales (1). Es importante anotar que todas las docentes se refieren a las manifestaciones de roles sexuales a ambientes o actividades observadas dentro de la institución, ya sea en el salón de clase o en las interacciones al aire libre.

\section{Cuadro 8 \\ Diferencia entre las niñas y los niños en el trabajo de aula, según opinión de las docentes.}

\begin{tabular}{lcc}
\hline Categoría de respuestas & $F$ & $\%$ \\
\hline Trabajo en áreas. & 14 & 70 \\
Conversación, rondas, filas. & 3 & 15 \\
Juegos al aire libre. & 1 & 5 \\
Aseo del aula. & 1 & 5 \\
No aplica. & 1 & 5 \\
\hline
\end{tabular}

Fuente: Elaboración propia.

En el cuadro ocho, ante la interrogante sobre la diferenciación entre niñas y niños en el trabajo de aula, las docentes responden en su mayoría (14) que la diferenciación se hace más palpable en el trabajo de áreas; por el contrario, tres de ellas consideran que se evidencia en el período de conversación, las rondas y la filas en las cuales se ubican de un lado a las niñas y del otro los niños. Una docente señala que los juegos al aire libre y el aseo del aula son los puntos básicos de la diferenciación. Otra docente no responde.

Es interesante notar que las docentes coinciden en que las diferencias en el trabajo de aula se dan en períodos colectivos, es decir, cuando las niñas y los niños interactúan entre sí. 
En términos generales, a partir de las opiniones de docentes de preescolar, acerca del sexismo, es posible señalar, entre otras cosas, lo siguiente:

- Las docentes de educación preescolar entrevistadas conciben género como una diferenciación en la que entran en juego varios aspectos como lo femenino y lo masculino, el sexo de cada persona y la cultura a la que pertenece; estos planteamientos son similares a lo que reporta la literatura.

- Los roles sexuales son percibidos por las docentes entrevistadas como un proceso conductual, en el que tanto el género, como la sociedad poseen un papel fundamental. Algunas de ellas opinan que los papeles sexuales son comportamientos y conductas de cada género mientras que otras aseguran que los roles sexuales obedecen a un patrón de conducta establecido en la sociedad en la que se encuentra inmersa la persona.

Es notorio cómo ambos planteamientos coinciden en que la conducta juega un papel crucial en la constitución de los roles sexuales, independientemente si estos son un asunto personal de cada género o, por el contrario, una herencia social aprendida.

- Las docentes también manifiestan que en la observación de los comportamientos de sus alumnos y alumnas, notan en sus interacciones conductas relacionadas con el sexismo; por ejemplo, manifiestan que dichas diferenciaciones las observan en los tipos de materiales y juegos que seleccionan las niñas y los niños; algunas docentes indican que el juego en las áreas es manifestación de roles sexuales y notan diferencias también en los juegos al aire libre.

- Por otra parte, las docentes coinciden en que las diferencias en el trabajo de aula se dan en periodos colectivos, es decir, cuando las niñas y los niños interactúan entre sí. También mencionan que cuando están en periodos de conversación o rondas, hay una evidente separación entre niños y niñas y entre los aspectos mencionados por ambos grupos.

- $\quad$ Se concluye además que para las docentes, las conductas sexistas de sus alumnos y alumnas obedecen a limitaciones de actuación producto de la influencia del hogar; señalan entre algunas de esas conductas: la minimización de la capacidad de la mujer o, por otro lado, la maximización de la capacidad del hombre en menosprecio de la capacidad femenina. Mencionan la diferenciación de los juegos en el jardín de niños. Es también un indicador importante el hecho de que algunas docentes se han abstenido de dar respuesta a algunas de las preguntas que versaban sobre diferencias entre alumnas y alumnos con carácter sexista. 
Por último, las conclusiones anteriores, se originaron como producto del análisis de instituciones educativas de nivel preescolar, las opiniones de docentes preescolares de dichas instituciones y de niños y niñas que cursaban ese nivel educativo. Por lo tanto, no son generalizables a la población costarricense, pero bien pueden constituirse en un valioso insumo para acercarse al análisis del sexismo en el aula preescolar y a la perspectiva de docentes preescolares acerca de dicho tema. 


\section{BIBLIOGRAFÍA}

Aguilar, L.; Barley, M.; Bertozzi,Y.; Calvo, Y.; Camac, E.; Camacho, L.; Escalante, A.; Facio, A.; González, M.; Gutiérrez, E.; Quirós, E.; y Trejos, M. (1995). ¿Feminismo en Costa Rica?. Testimonios, Reflexiones; Ensayos. San José, Costa Rica: Mujeres.

Ayales, I.; Benítez, M.; Fevrier, S. y Ramírez, I. (s.f.). Género, comunicación y desarrollo sostenible: Aportes conceptuales y metodológicos. San José, Costa Rica: IICA.

Castro, S. (1999). Socialización diferenciada por género. San José, Costa Rica: EDILOY.

Barrantes, R. (2000). Investigación: un camino al conocimiento, un enfoque cuantitativo y cualitativo. San Jose: Editorial de la Universidad Estatal a Distancia.

González, M. (1990). El sexismo en la educación: la discriminación cotidiana. San José, Costa Rica: Editorial Universidad de Costa Rica.

Lagarde, M. (1999). Una mirada feminista en el umbral del milenio. Heredia. Costa Rica: Instituto de Estudios de la Mujer.

Luna, I. (2003). Andrógina y Postmodernismo: Una aproximación desde la Psiquiatría. En línea.Disponible: http://www.psiquiatria.com/interpsiquis2003/9996

Quirós, Y. (1989). Sistemas de valores y percepción de los roles sexuales en dos sectores de mujeres profesionales costarricenses. Tesis no publicada. Universidad de Costa Rica.

Scala, J. (2001). Género y Derechos humanos. $1^{\text {a }}$ ed. San José, Costa Rica. Ediciones PROMESA. 


\section{GLOSARIO}

Sexismo: (...) se denomina sexismo a todos aquellos procesos que limitan el desarrollo de las potencialidades esenciales debido al sexo de cada persona y por ende de su grupo de iguales (...), el sexismo es la discriminación de individuos e individuas relacionada con la pertenencia a un sexo determinado (González, 1990, p. 11).

Roles o papeles sexuales: están relacionados con el comportamiento de los seres humanos de acuerdo con el sexo al que pertenecen, a saber: femenino o masculino, respondiendo esto a un tipo de sociedad determinada. Estos son fortalecidos tanto en la familia, como en la escuela y, de ese modo, se incorporan a la conducta de cada persona.

Docentes: para efectos de esta investigación, se contó con la participación de un total de 20 docentes, que laboraban en los jardines infantiles públicos. Las instituciones fueron escogidas por los criterios de acceso y disponibilidad de recursos. Cabe destacar, que de cada institución se escogieron cuatro docentes, que laboraban en educación preescolar y algunas específicamente en el ciclo de Transición.

Niños y niñas: para este estudio, se contó con la participación de 60 estudiantes de 5 a 6 años de edad, que asistían al jardín infantil público. Los niños (30) y las niñas (30) fueron seleccionados al azar. 